\title{
Single Domain Switching Investigated Using Telegraph Noise Spectroscopy: Possible Evidence for Macroscopic Quantum Tunneling
}

\author{
F. Coppinger, ${ }^{1,2}$ J. Genoe, ${ }^{1}$ D. K. Maude, ${ }^{1}$ Ulf Gennser, ${ }^{1, *}$ and J. C. Portal ${ }^{1,2}$ \\ ${ }^{1}$ High Magnetic Field Laboratory-Centre National de la Recherche Scientifique, BP 166, 38042 Grenoble, France \\ ${ }^{2}$ Institut National des Sciences Appliquées, 31077 Toulouse, France \\ K. E. Singer, P. Rutter, T. Taskin, A. R. Peaker, and A. C. Wright ${ }^{\dagger}$ \\ Department of Electrical Engineering and Electronics and Centre for Electronic Materials, \\ University of Manchester Institute of Science and Technology, Sackville Street, Manchester, M60 1QD, United Kingdom
}

(Received 6 April 1995)

\begin{abstract}
Telegraph noise, i.e., two-level fluctuations (TLF), in the magnetoresistance of Er-doped GaAs has been used to probe the magnetic moment of the small ErAs clusters formed during the molecular beam epitaxy growth process. At high temperatures the TLF are thermally activated but below $350 \mathrm{mK}$ tunneling of the magnetization dominates.
\end{abstract}

PACS numbers: 73.50.Td, 73.40.Gk, 75.70.Kw

Recent theoretical papers [1] have led to an increased interest in quantum tunneling of magnetization. In small single domain (SD) magnetic particles such a process is referred to as macroscopic quantum tunneling (MQT). The magnetic moment of a ferromagnetic SD particle has been measured using a dc SQUID microsusceptometer capable of detecting the magnetic field produced by as few as $10^{6}$ electron spins [2]. However, in ferromagnetic particles the barrier between the two equivalent macroscopic spin configurations is large, and thus the tunneling probability of the magnetization is low. It has been suggested that SD antiferromagnetic particles, which have a nonzero magnetic moment due to the uncompensated spins at the surface, are better candidates for the observation of MQT because of the smaller energy barrier separating the two macroscopic spin configurations [3].

In this Letter, we use telegraph noise spectroscopy to directly probe the magnetic moment of extremely small ErAs clusters in a matrix of GaAs. The telegraph noise or two-level fluctuations (TLF) is observed in the resistance of a sample containing self-organizing ErAs quantum wires and dots in a semi-insulating GaAs matrix. We show that the TLF are related to two possible magnetic states of an ErAs cluster and that the energy difference between the two states is a linear function of the magnetic field, as expected for states with different magnetic moments. This technique is capable of measuring a magnetic moment corresponding to a few tens of electron spins, many orders of magnitude below that possible with state of the art dc SQUID microsusceptometers. At high temperatures $(0.35-2 \mathrm{~K})$ the telegraph noise is thermally activated over more than 2 orders of magnitude. However, when the energy barrier separating the two states is minimized by rotating the sample in situ, the transition rate at low temperature $(<350 \mathrm{mK})$ is orders of magnitude larger than expected, which we interpret as evidence for the tunneling of magnetization.
Thin layers of semimetallic ErAs grown on GaAs by molecular beam epitaxy (MBE) remain semimetallic [4] despite theoretical predictions that confinement effects should open up a semiconducting gap. Such layers of ErAs are antiferromagnetic at low temperatures and low magnetic field but an antiferromagnetic-paramagnetic phase transition is induced with increasing temperature or magnetic field [5]. With appropriate conditions during the MBE growth of Er-doped GaAs the Er precipitates as ErAs to form dots or wires with a diameter of a few nanometers $[6,7]$.

For the investigation of such ErAs precipitates, $n-i-n$ mesa structures were fabricated using MBE. A $1 \mu \mathrm{m}$ thick Se-doped $\left(N_{d}=10^{18} \mathrm{~cm}^{-3}\right)$ layer was grown on a (100) $n^{+}$substrate, followed by a $25 \mathrm{~nm}$ thick GaAs layer with the same Se concentration but with an additional $\mathrm{Er}$ doping of $3 \times 10^{19} \mathrm{~cm}^{-3}$. $50 \mathrm{~nm}$ of GaAs: $\operatorname{Er}$ (Er concentration $=3 \times 10^{19} \mathrm{~cm}^{-3}$ ) was grown with no intentional $n$-type doping. Identical GaAs:(Se,Er) and GaAs:Se layers were subsequently grown to form the top contact. Under the growth conditions used (see Ref. [7]) the ErAs aggregates into very small interconnected quantum wires and quantum dots. Cross-sectional transmission electron microscopy [7] shows that the ErAs wires and dots, embedded in the GaAs, have an average diameter of $3 \mathrm{~nm}$. Mesa structures, of size $10 \times 15 \mu \mathrm{m}^{2}$, were fabricated using conventional photolithography and wet etching techniques. Ohmic contacts were formed with alloyed AuGe/Ni. All resistance measurements were performed by applying an ac current (10-30 nA) perpendicular to the layers.

The magnetoresistance of a typical mesa, with the magnetic field $(H)$ parallel to the current at a temperature of $300 \mathrm{mK}$, is shown in Fig. 1. A large negative magnetoresistance is observed with a temperature dependence (not shown) qualitatively similar to that observed in the longitudinal magnetoresistance in ErAs thin films, and attributed to the spin disorder scattering [5]. Superimposed on this 


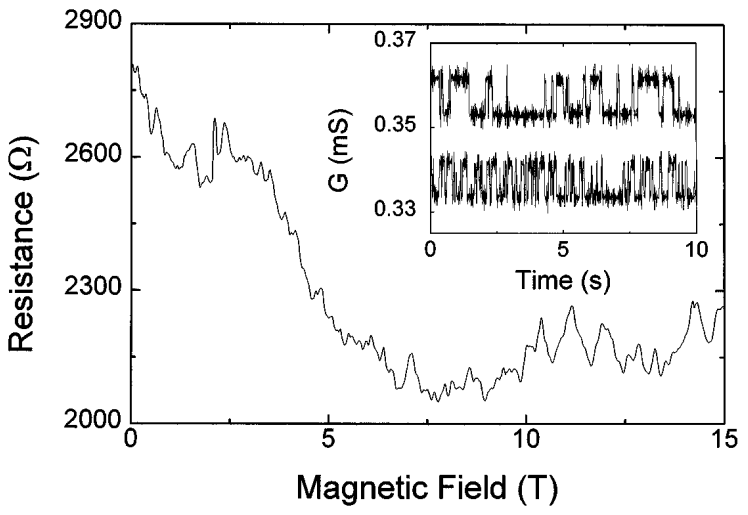

FIG. 1. Magnetoresistance showing the universal conductance fluctuations measured at $300 \mathrm{mK}$. The inset shows the telegraph noise observed at a fixed magnetic field of $0 \mathrm{~T}$ and at temperatures of 1.72 (top) and $1.90 \mathrm{~K}$ (bottom).

negative magnetoresistance background are universal conductance fluctuations (UCF), which arise from the interference of the partial electron wave functions as they traverse the structure. A phase-coherent length $L_{\Phi} \sim 200 \mathrm{~nm}$ has been deduced from the typical period of the UCF. It is not clear whether the electronic properties are dominated by transport through the wires or through the undoped GaAs matrix. The amplitude of the UCF indicates that the resistivity is dominated by a few phase-coherent subsystems in parallel, and a percolation picture is therefore appropriate to describe electronic conduction through the device [8].

Moreover, superimposed on these fluctuations are instabilities (sudden jumps) in the magnetoresistance. The instabilities are observed only at low temperature $(T<$ $3 \mathrm{~K})$ and moderate magnetic field $(H<4 \mathrm{~T})$. Their amplitude is of the order of the amplitude of the UCF, indicating that the resistance is dominated by only a few small phase-coherent subsystems of lateral size $L_{\Phi}$. The jumps in the resistance are due to a change in the magnetic moment of a small domain of ErAs during the magnetic field sweep, which changes the electron scattering. The phase change caused by this scattering will affect the value of the total resistance, because of the phase coherence of the system. We have occasionally seen three-level systems (not shown) that display hysteresis. Two-level systems are more often observed, and over a certain magnetic field region the resistance exhibits telegraph noise, i.e., two discrete values as a function of time (see inset of Fig. 1).

Telegraph noise or TLF have been extensively studied in metal nanostructures and tunnel junctions and can be used to probe the nature of the impurity responsible $[9,10]$. In our investigation, the power spectra $S(f)$ of the TLF and the histograms of both switching-time distributions prove that the switching between the states is uncorrelated. The average time spent in each state is directly calculated throughout this paper by averaging over a large number of switching events. The dynamics of the system can be modeled as two states separated by a barrier. The switching is thermally activated and follows the

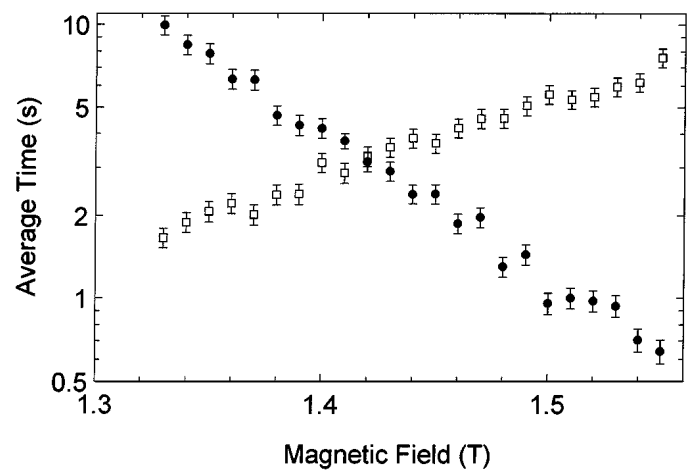

FIG. 2. Average time spent in the low ( $\square$ ) and high ( resistance states as a function of magnetic field for a typical telegraph noise measured at $1.5 \mathrm{~K}$.

law $\tau_{i}=\tau_{0, i} \exp \left(E_{i} / k T\right)$, where $E_{i}$ represents the height of the barrier separating the higher or lower state. In Fig. 2 it can be seen that the average time spent in the higher and lower states varies exponentially with the magnetic field. The ratio of the average times spent in the two states gives a direct access to the energy difference $(\Delta E)$ between these states. The validity of this model has been verified by sweeping the temperature for a number of different magnetic fields. The TLF $a-c$ were thermally activated with an activation energy in the range $12-22 \mathrm{~K}$. The ratio of the average time was also thermally activated within experimental error, meaning that the energy difference between the two states is independent of temperature and the ratio $\tau_{0, \text { high }} / \tau_{0, \text { low }}$ is independent of magnetic field. This enables us to determine $\Delta E=k T \ln \left(\tau_{\text {high }} / \tau_{\text {low }}\right)$ at a fixed temperature (Fig. 3). The difference in energy between the two states varies linearly with magnetic field and is symmetric about $H=0$ [11]. The role of the magnetic field is to tune the energy of the two states so that they are almost identical. Under these conditions switching can occur via a thermally activated process. At other magnetic fields, the energy difference between the states is so large, that one of the states is dominant and no switching events are observable on an experimentally realistic time scale.

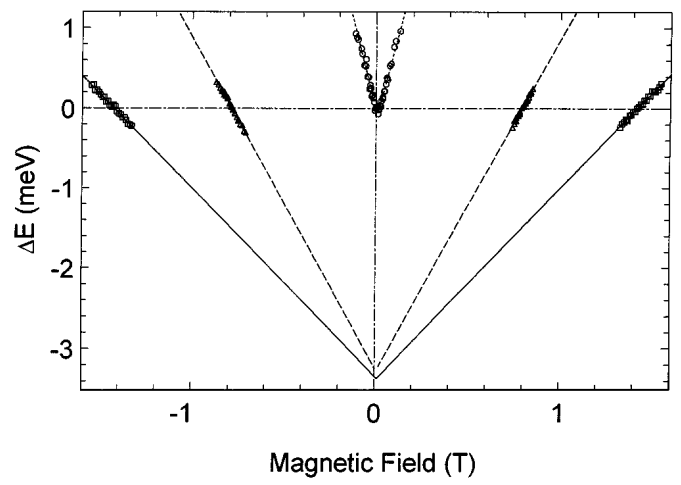

FIG. 3. Energy difference between the two magnetic states responsible for the TLF $(a-c)$. The solid and dashed lines are linear fits. These three different TLF shown here were measured after thermally cycling the sample to room temperature, which allows us to investigate different switchers. 
TABLE I. The difference of magnetic moment (projected onto the magnetic field axis and in units of $\mu_{B}$ ) between the well state and the virtual barrier state for the low and high resistance states.

\begin{tabular}{lcccc}
\hline \hline & \multicolumn{2}{c}{$H<0$} & \multicolumn{2}{c}{$H>0$} \\
\multicolumn{1}{c}{ Resistance } & High & Low & High & Low \\
\hline TLF $a(0 \mathrm{~T})^{\mathrm{a}}$ & $104 \pm 9$ & $-50 \pm 8$ & $113 \pm 5$ & $-47 \pm 9$ \\
TLF $b( \pm 0.8 \mathrm{~T})^{\mathrm{a}}$ & $-30 \pm 0.6$ & $+41 \pm 0.7$ & $-29 \pm 1$ & $+44 \pm 1.1$ \\
TLF $c( \pm 1.4 \mathrm{~T})^{\mathrm{a}}$ & $+26 \pm 0.6$ & $-14 \pm 0.5$ & $+27 \pm 0.6$ & $-14 \pm 0.6$ \\
TLF $d(1.7 \mathrm{~T})^{\mathrm{b}}$ & Not investigated & $+34 \pm 1.2$ & $+50 \pm 1.7$ \\
TLF $e(1.61 \mathrm{~T})^{\mathrm{b}}$ & Not investigated & $-53 \pm 1.4$ & $+37 \pm 1.4$ \\
\hline \hline
\end{tabular}

aMagnetic fields $H \|[100]$ (growth direction).

${ }^{\mathrm{b}}$ Measured under rotation in situ (values for $H \|[011]$ ).

Zimmerman, Golding, and Haemmerle [12] have previously studied TLF associated with nonmagnetic impurities and observed a random dependence of $\Delta E$ with magnetic field, which they attributed to a coupling between the local carrier density and the defect. In our case, the linear dependence is due to the magnetic properties of ErAs. The difference in the magnetic moment of the two states leads to an energy difference $\Delta E=\Delta m H$, where $\Delta m$ is the difference in the projection of the magnetic moment on the magnetic field axis $H$. From the slope of the linear dependence shown in Fig. 3, it is possible to extract the value of $\Delta m$. These values are $152 \mu_{B}, 71.5 \mu_{B}$, and $41 \mu_{B}$ for the TLF observed at $0,0.8$, and $1.4 \mathrm{~T}$, respectively (see Table I). The magnetic moment carried by one Er atom in a ErAs crystal lies between $5 \mu_{B}$ and $8 \mu_{B}$ [13]. This suggests that a few tens of Er atoms are involved in the spin-flip process. However, it is not possible to extract directly the number of Er atoms involved in these transitions because $\Delta m$ represents the projection of the magnetic moment carried by the two states on to the magnetic field axis.

The linear dependence shows that the energy splitting is controlled by the magnetic field so the average time spent in one state can be written as $\tau_{i}=\tau_{0, i} \exp \left[\left(E_{i}+m_{i} H-\right.\right.$ $\left.\left.m_{b} H\right) / k T\right]$, where $E_{i}$ is the activation energy independent of the magnetic field, $m_{i}$ the projection of the magnetic moment carried by the state $i$ on the magnetic field axis, and $m_{b}$ the projection of the magnetic moment of the virtual state in the barrier on the magnetic field axis. This means that $m_{i}-m_{b}$ can be directly deduced from the slope of a $k T \ln \left(\tau_{i}\right)$ vs $H$ plot. The values of $m_{i}-m_{b}$ of the TLF studied in this paper are given in Table I. All the states have a small but nonzero magnetic moment as expected for small antiferromagnetic clusters.

ErAs crystallizes in the $\mathrm{NaCl}$ structure [7]. The magnetic moments are all ferromagnetically coupled along the (111) plane, and have an antiferromagnetic coupling between these planes [5]. In small antiferromagnetic particles the magnetic moment of the two sublattices is not completely compensated due to statistical fluctuations in the direction of the magnetic moment of the magnetic atoms at the surface [3]. For a small cluster of ErAs of $3 \mathrm{~nm}$ diameter the uncompensated magnetic moment due to statistical fluctuations at the surface would be $\sim 50 \mu_{B}$, which is of the same order of the magnetic moment carried by all our states (see Table I).

It has been suggested that antiferromagnetic clusters are better candidates to see macroscopic quantum tunneling than ferromagnetic compounds [3]. In our system the attempt time $\tau_{0}$ for traversing the barrier between the two states is measured to be of the order of $10^{-6}-10^{-3} \mathrm{~s}$, somewhat larger than that measured for small cobalt particles $10^{-6}$ [13]. Rotating the sample in situ in the dilution refrigerator allows us to probe the anisotropy of the energy barrier separating the two states. The energy barrier varies between 4 and $12 \mathrm{~K}$ for the TLF investigated (TLF $d$ and $e$ in Table I). These measurements will be presented in detail elsewhere. We limit ourselves here to
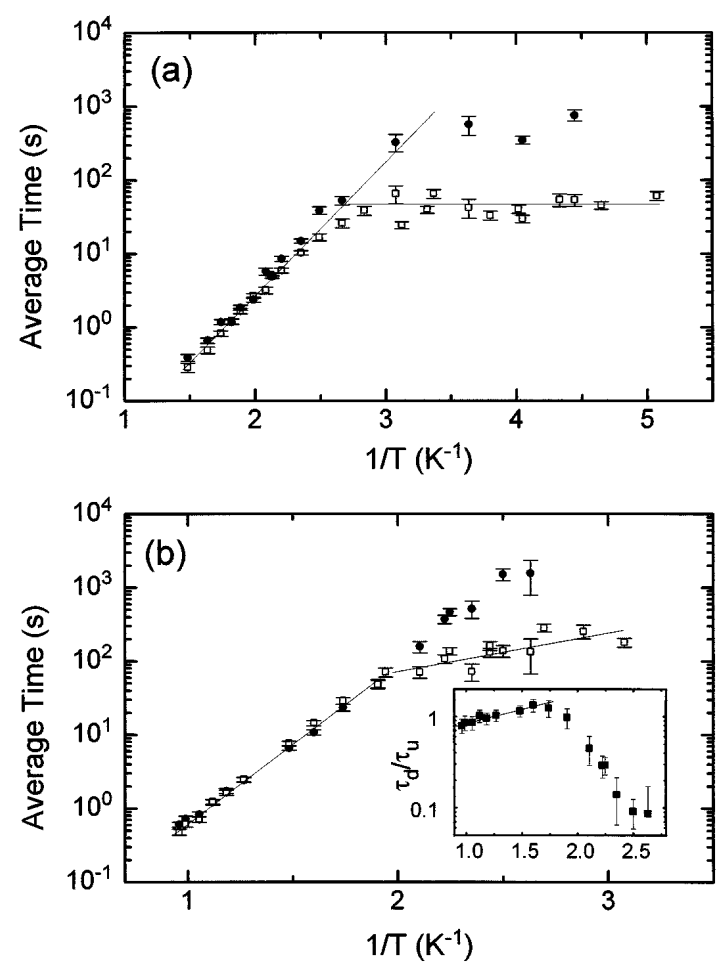

FIG. 4. Average time spent in the low ( $\square$ ) and high ( resistance states as a function of inverse temperature for TLF $d$ at $1.70 \mathrm{~T}$ (a) and for TLF $e$ at $1.61 \mathrm{~T}$ (b). The solid lines are linear fits to the thermally activated and tunneling portions of the curves. The inset of (b) shows the ratio of both times indicating that detailed balance is not observed. 
TABLE II. Parameters of the tunneling process (critical temperature $T_{c}$ and dwell time $\tau_{\text {dwell }}$ ) and of the thermally activated process (activation energy $E_{\text {act }}$ and $\tau_{0}$ ).

\begin{tabular}{lclcccc}
\hline \hline & Field $(\mathrm{T})$ & State & $T_{c}(\mathrm{mK})$ & $\tau_{\text {dwell }}(\mathrm{s})$ & $E_{\text {act }}(\mathrm{K})$ & $\tau_{0}(\mathrm{~s})$ \\
\hline TLF $d^{\text {a }}$ & 1.70 & Low & 375 & 50 & 4.13 & $6 \times 10^{-4}$ \\
& & High & 315 & 520 & 4.52 & $4 \times 10^{-4}$ \\
TLF $d$ & 1.68 & Low & 360 & 16.5 & Not investigated \\
TLF $e^{\text {a }}$ & 1.61 & Low & 500 & 156 & 5.24 & $3 \times 10^{-3}$ \\
& & High & & & 4.69 & $7 \times 10^{-3}$ \\
TLF $e$ & 1.58 & Low $^{\mathrm{b}}$ & 470 & 65 & Not investigated \\
TLF $e$ & 1.56 & Low $^{\mathrm{b}}$ & 445 & 33 & Not investigated \\
\hline \hline
\end{tabular}

a Determined from experimental data shown in Fig. 4.

${ }^{\mathrm{b}}$ The high state is not measurable on a realistic time scale.

the configuration in which the experimentally measured energy barrier is a minimum. The temperature dependence of the average time spent in the high and low resistance states in this minimum energy configuration is shown in Fig. 4 for TLF $d(1.70 \mathrm{~T})$ and TLF $e(1.61 \mathrm{~T})$. The obtained average time is thermally activated over 2 orders of magnitude at high temperatures. Below $350 \mathrm{mK}$, this average time for the low (high) state deviates. At the lowest temperatures investigated, the average time in the low state is 5 orders of magnitude less than expected for a thermally activated process, giving evidence for the observation of MQT. The results are summarized in Table II. The inset of Fig. 4(b) reveals that detailed balance is not preserved in the tunneling regime, in contrast with experiments on tunneling in two-level systems coupled with a bath of electrons in metallic systems [14]. We stress that this is not due to a "hang-up" in the temperature because one of the states continues to be thermally activated, clearly demonstrating that the ErAs cluster is in equilibrium with the fridge. In our semiconductor samples, with orders of magnitude lower electron density, it is probably the coupling with the bath of nuclear spins that is the dominant dissipative mechanism.

In summary, we have observed TLF in the resistance of a self-organizing network of semimetallic ErAs wires and clusters in a matrix of GaAs. The switching is due to the change in the orientation of the magnetic moment of a few tens of Er atoms in a small cluster of ErAs. Telegraph noise spectroscopy has been shown to be a very powerful tool to probe the magnetic properties of a small cluster of magnetic material. At low temperature, a deviation from the TA behavior is observed and attributed to MQT. Further work is required to understand the nonpreservation of detailed balance and confirm the observation of MQT.

This research is supported through ESPRIT BRA III (7260) and the Conseil Régional de Midi-Pyrénées. We thank P. Stamp and B. Barbara for useful discussions.

*Present address: Paul Scherrer Institut, Villigen, Switzerland.
${ }^{\dagger}$ Also at AMRL, N.E. Wales Institute, Deeside College, Connah's Quay, CH5 4HR, Wales.

[1] For a review, see P.C. E. Stamp, E. M. Chudnovsky, and B. Barbara, Int. J. Mod. Phys. B 6, 1355 (1992).

[2] D. D. Awschalom, J. R. Rozen, M. B. Ketchen, W. J. Gallagher, A. W. Kleinsasser, R. L. Sandstrom, and B. Bumble, Appl. Phys. Lett. 53, 2108 (1988).

[3] B. Barbara and E. M. Chudnovsky, Phys. Lett. A 145, 205 (1990).

[4] S. J. Allen, Jr., N. Tabatabaie, C. J. Palmstrøm, S. Mounier, G. W. Hull, T. Sands, F. DeRosa, H. L. Gilchrist, and K. C. Garrison, Surf. Sci. 228, 13 (1990).

[5] S. J. Allen, Jr., N. Tabatabaie, C. J. Palmstrøm, G. W. Hull, T. Sands, F. DeRosa, H. L. Gilchrist, and K. C. Garrison, Phys. Rev. Lett. 62, 2309 (1989).

[6] I. Poole, K. E. Singer, A. R. Peaker, and A.C. Wright, J. Cryst. Growth 121, 121 (1992).

[7] K.E. Singer, P. Rutter, A. R. Peaker, and A.C. Wright, Appl. Phys. Lett. 64, 707 (1994).

[8] F. Coppinger, D. K. Maude, U. Gennser, J.C. Portal, K. E. Singer, P. Rutter, A.R. Peaker, and A.C. Wright, Proceedings of the ICPS-22 (World Scientific, Singapore, 1995), Vol. 2, p. 1791.

[9] P. M. Campbell, E.S. Snow, W.J. Moore, O. J. Glembocki, and S. W. Kirchoefer, Phys. Rev. Lett. 67, 1330 (1991).

[10] Xiuguang Jiang, M. A. Dubson, and J.C. Garland, Phys. Rev. B 42, 5427 (1990).

[11] All magnetic potential anisotropies (crystalline, form, stress, etc.), apart from the remanent field from surrounding clusters and the external applied magnetic field, deliver a potential for the giant spin with a symmetry such that if there is a minimum under a magnetic field $H$ at the angle $\theta$, there is also a minimum under a magnetic field $-H$ at the angle $\theta+\pi$. The spin clusters switch to the opposite direction when the magnetic field is reversed.

[12] N. M. Zimmerman, B. Golding, and W.H. Haemmerle, Phys. Rev. Lett. 67, 1322 (1991).

[13] W. Wernsdorfer, K. Hasselbach, D. Mailly, B. Barbara, A. Benoit, L. Thomas, and G. Suran, J. Magn. Magn. Mater. 145, 33 (1995).

[14] B. Golding, N. M. Zimmerman, and S. N. Coppersmith, Phys. Rev. Lett. 68, 998 (1992). 